\title{
PERCEPCIONES Y MANIFESTACIONES DE DISCRIMINACIÓN EN EL ÁMBITO UNIVERSITARIO. UN PUNTO DE PARTIDA PARA SU ERRADICACIÓN ${ }^{1}$
}

\section{PERCEPTIONS AND MANIFESTATIONS OF DISCRIMINATION IN THE UNIVERSITY CONTEXT. A STARTING POINT FOR ITS ERADICATION}

\author{
Silvia Jiménez Mata* \\ Sileny Mena Gómez ** \\ María Luisa Preinfalk Fernández***
}

\section{RESUMEN}

Este artículo se basa en una investigación sobre el análisis de las formas de discriminación por género, etnia-raza, orientación del deseo sexual y discapacidad, en el ámbito universitario. Se efectuó durante los años 2017-2019, con el objetivo de conocer las percepciones del estudiantado de la Universidad Nacional de Costa Rica. Se utilizó un enfoque mixto de investigación por medio de la realización de grupos focales y la aplicación de un cuestionario a una muestra probabilística de estudiantes, con lo cual se evidenció un alto grado de manifestaciones de discriminación.

PALABRAS CLAVE: DISCRIMINACIÓN * UNIVERSIDAD * GÉNERO * ETNIA * DISCAPACIDAD

$1 \quad$ Este trabajo corresponde a los resultados del proyecto: "Percepciones y manifestaciones de discriminación: un estudio en la comunidad estudiantil de la Universidad Nacional”, desarrollado por el Instituto de Estudios de la Mujer de la Universidad Nacional de Costa Rica.

* Instituto de Estudios de la Mujer de la Universidad Nacional, Costa Rica. silvia.jimenez.mata@una.cr

** Instituto de Estudios de la Mujer de la Universidad Nacional, Costa Rica. sileny.mena.gomez@una.cr

*** Instituto de Estudios de la Mujer de la Universidad Nacional, Costa Rica. maria.preinfalk.fernandez@una.cr 


\section{ABSTRACT}

This article is based on an investigation carried out during the years of 2017 and 2019 at the National University of Costa Rica. The research aimed to identify the perceptions of the students concerning the forms of discrimination related to gender, ethnic-race, sexual orientation and disability in the university environment. Hence, a mixed research approach was used, through the application of a questionnaire to a probabilistic sample of students and through the conformation of focus groups, all of which evidenced a high degree of manifestations of discrimination.

KEYWORDS: DISCRIMINATION * UNIVERSITY * GENDER * ETHNICITY * DISABILITY

\section{INTRODUCCIÓN}

Las diversas formas de discriminación presentes en la sociedad costarricense descansan en un sistema patriarcal y capitalista que establece estrategias de diferenciación y exclusión hacia las personas, con base en una serie de características individuales y sociales.

Las instancias de educación superior, al estar insertas en este sistema patriarcal, juegan un papel importante, tanto en la reproducción como en el cuestionamiento de prácticas sociales dentro del espacio universitario que pueden resultar discriminatorias. Por esta razón, la Universidad Nacional (UNA), desde el 2011, mediante el Acuerdo del Consejo Universitario scu-1519-2011, se declaró como un "Espacio libre de todo tipo de discriminación", por razones de diversidad de géneros, orientación sexual, pertenencia étnica y de clases sociales. En el año 2015, se acordó ampliar la declaratoria, mediante Acuerdo del Consejo Universitario UNA-SCU-ACUE-1313-2015, incluyendo la discriminación por razones de sexo, identidad de género, discapacidad, edad, creencia religiosa, apariencia o lugar de procedencia.

En el marco de los acuerdos referidos se llevó a cabo el proyecto de investigación: "Percepciones y manifestaciones de discriminación: Un estudio en la comunidad estudiantil de la Universidad Nacional", del cual se desprende el presente artículo. Dicho proyecto se desarrolló durante el periodo de enero de 2017 a junio de 2019, con el objetivo de analizar las manifestaciones de discriminación según género, etnia-raza, discapacidad y orientación del deseo sexual presentes en el ámbito universitario de acuerdo con la percepción del estudiantado de la UNA.
Anteceden a este proyecto una serie de acciones implementadas en la UNA, que buscan garantizar el acceso, la permanencia y la graduación de estudiantes que provienen de poblaciones vulnerabilizadas, tales como: el Programa de Becas, el Sistema Estratificado de Admisión, la Comisión Institucional en Materia de Discapacidad (CIMAD), el Programa UnA Educación de Calidad, el Programa Éxito Académico, entre muchas otras iniciativas. Destaca como una acción afirmativa que ha dado resultados importantes, la impulsada desde la División de Educación Rural (DER) del Centro de Investigación y Docencia en Educación (CIDE), de eximir del examen de admisión a estudiantes provenientes de pueblos indígenas, así como, el traslado de docentes a comunidades rurales para impartir lecciones como una medida para promover el ingreso y la graduación de población indígena en la carrera de Educación con énfasis en Educación Rural I y II ciclos.

Estos esfuerzos muestran el compromiso de la una por constituirse en una casa de estudios inclusiva, por lo que se espera que los resultados de este estudio no solo contribuyan a identificar las principales formas de discriminación presentes en la vida universitaria, sino también a elaborar estrategias y aplicar acciones que garanticen un ambiente universitario libre de toda forma de discriminación.

\section{PERSPECTIVAS TEÓRICAS PARA COMPRENDER LAS DISCRIMINACIONES}

Para acercarse a la comprensión de las discriminaciones presentes a nivel social y, específicamente, en las instituciones educativas, 
se parte de la prevalencia del patriarcado y del capitalismo, entendidos como sistemas económicos, culturales y políticos que operan en forma articulada. Si bien, dichos sistemas se han transformado a lo largo de la historia $y$ operan en forma diferenciada en cada contexto social-cultural, se puede apuntar que se han caracterizado por reproducir mecanismos de exclusión y dominio, en razón de factores como lo son el sexo, las identidades de género, la clase social, la condición étnica racial, la discapacidad, entre otros.

Las ideologías, entendidas como "...sistemas coherentes de creencias que orientan a las personas hacia una manera concreta de entender $y$ valorar el mundo; proporcionan una base para la evaluación de los acontecimientos, las conductas y otros fenómenos sociales; $y$ les sugieren respuestas de comportamiento..." (Saltzman, 1992, p.44); tienen un papel fundamental en la reproducción de las relaciones de poder y permean las principales instituciones sociales como lo son las familias, el sistema educativo, las instituciones laborales y los Estados.

Se parte de un enfoque de género interseccional, desde el cual se reconoce que persisten a nivel social estructuras de exclusión como lo son el sexismo, el racismo, el clasismo, la homofobia y el capacitismo, las cuales tienen como base factores culturales, económicos y políticos, que operan como mecanismos ideológicos y materiales en el marco del patriarcado y del capitalismo. El carácter interseccional radica en que dichas estructuras operan en forma articulada, lo que se traduce en exclusiones múltiples para quienes son ubicados o ubicadas según sus características físicas y fenotípicas y sus identidades en las categorías que son desvalorizadas o inferiorizadas en el marco de dichos sistemas de exclusión (Munévar y Mena, 2009 y 2013).

En la Convención Interamericana contra toda forma de Discriminación e Intolerancia, firmada por Costa Rica en abril de 2019 (OEA, 2019), se entiende por discriminación cualquier acto de “...distinción, exclusión, restricción o preferencia, en cualquier ámbito público o privado, que tenga el objetivo o el efecto de anular o limitar el reconocimiento, goce o ejercicio, en condiciones de igualdad, de uno o más derechos humanos" (art.1).

A partir de dicha definición normativa, se establece que la discriminación en cualquiera de sus manifestaciones $y$ tanto si es ejercida en forma directa como indirecta contra una persona, constituye una falta grave en la medida en que se limita la garantía de los derechos humanos, así como, el principio de igualdad ante la Ley.

Las opresiones/exclusiones múltiples se manifiestan en formas de discriminación dirigidas a personas cuyos atributos físicos e identitarios son desvalorizados, en el marco de un sistema social que establece dicotomías que jerarquizan, $y$ que por tanto, refuerzan las relaciones de poder imperantes en un momento dado. Como se señaló, las discriminaciones tienen bases estructurales en los sistemas patriarcales y capitalistas, en los cuales se legitima y refuerza, desde todas las instituciones sociales, prejuicios asociados al sexismo, a la homofobia, al racismo y al capacitismo.

Toda acción de discriminación tiene como trasfondo prejuicios y estereotipos que buscan justificar la exclusión de ciertas personas o grupos, asumiendo que las diferencias respecto al grupo que discrimina son las que provocan la exclusión y el rechazo, y no el acto en sí mismo. Se entiende por estereotipo a las cogniciones $y$ pensamientos que evalúan como positivas o negativas las características de un grupo o persona; mientras que el prejuicio hace referencia a la acción o a los afectos negativos hacia personas o grupos, generalmente a partir de experiencias afectivas previas o por la referencia de otras personas, sobre quien es discriminada (Puertas, 2004).

En el contexto de sociedades patriarcales opera la construcción social y cultural de la diferencia sexual, lo que implica que cada cultura otorga significados al ser mujer, al ser hombre $y$ en algunos contextos a personas que no se identifican con dichas categorías. Estos significados tienen un alcance mayor en la medida en que impactan y regulan otros ámbitos sociales, como lo son: el trabajo, la educación, la familia y las comunidades. Siguiendo a Lamas (2002), "Esta simbolización cultural 
de la diferencia anatómica toma forma en un conjunto de prácticas, ideas, discursos y representaciones sociales que dan atribuciones a la conducta objetiva y subjetiva de las personas en función de su sexo" (pp.57-58).

Paralelamente a las diferenciaciones que se establecen a nivel social entre los sexos, opera una jerarquización de las conductas, roles e identidades asociados a hombres y mujeres, donde aquellos atribuidos a los primeros son valorados como superiores, mientras que los otorgados a las mujeres son considerados de menor valía. Lo anterior se expresa en el sexismo presente en diferentes ámbitos como lo son el lenguaje, los trabajos realizados, e inclusive, a nivel de las normativas de las instituciones que tienen como referente exclusivamente a los hombres como sujetos sociales.

El capitalismo patriarcal, desde sus inicios a finales del siglo xvIII, estableció al hombre blanco de clase media $y$ heterosexual como referente de lo humano. Dicho modelo familiar suponía además el rol maternal y de amas de casa delegado en las mujeres, fenómeno que se dio específicamente en los sectores medios. De esta forma, se refuerza la división sexual del trabajo, estableciendo una marcada brecha entre el ámbito público (producción) y el ámbito privado (reproducción). Dicha estructuración de la familia moderna resultaba funcional al sistema capitalista, en la medida en que se aseguraba la reproducción de la fuerza de trabajo y la complementariedad entre los sexos (Meler, 1998).

Por lo tanto, los sistemas económicos y políticos dominantes regulan además el campo de la sexualidad, entendida como "...una elaboración psíquica y cultural de los placeres en los intercambios corporales (construida discursivamente, regulada y reglamentada mediante prohibiciones y sanciones que le dan, literalmente, forma $y$ direccionalidad)..." (Lamas, 2002, p.61).

Una de las principales regulaciones que en el marco de sociedades occidentales se ha establecido a partir del siglo xviII, es el establecimiento de la heterosexualidad como la forma "natural" y legítima de ejercicio de la sexualidad, generando una serie de prejuicios, entre los que se encuentran, la homofobia, la lesbofobia, la bifobia, la transfobia, entre otros, y que se expresa en un rechazo hacia las personas que tienen orientaciones, identidades $y$ prácticas sexuales que transgreden la heterosexualidad. A nivel social persisten normas y leyes que limitan la igualdad de derechos civiles y patrimoniales para dichas personas, lo cual da cuenta del impacto de la discriminación.

Otro sistema dominante que ha operado históricamente en forma articulada al capitalismo y al patriarcado es el racismo, el cual de acuerdo con Campbell (2002), se define como "...un fenómeno ideológico y una doctrina que se basa en las diferencias raciales para fundamentar la supremacía de la raza blanca sobre las demás" (p.24).

Segato (2006) expone que el racismo parte de la interpretación negativa hacia el aspecto físico de los pueblos y la atribución en forma automática y prejuiciosa de características intelectuales y morales, en donde todo lo valorado positivamente se relaciona con las personas que se asumen blancas, mientras que lo negativo y rechazado se instaura en la gente excluida, las personas pensadas como no blancas.

El autor agrega que existen diferentes víctimas del racismo, ya sea por discriminación de "raza con etnia", donde confluye la discriminación a partir de una diferencia racial o un signo fenotípico con un patrimonio cultural idiosincrático; la discriminación de "raza sin etnicidad", excluyendo a quienes comparten trazos raciales (color de piel, forma del cabello, labios o nariz), pero no comparten un patrimonio cultural como tal; $y$ la discriminación por "etnicidad sin raza", discriminándose aquellas personas que conciben como propio el patrimonio cultural del pueblo de pertenencia, pero no presentan trazos raciales que les distingan como parte de esa población.

Es importante apuntar que tanto la discriminación étnica como racial está articulada al sexismo, lo que implica que las mujeres enfrentan una doble discriminación por el hecho de ser afrodescendientes o indígenas, $y$ por su condición de género. Desde un análisis de género interseccional cobra especial relevancia el análisis de las formas que adquieren dichas opresiones en contextos históricos dados, con el fin de no caer en posiciones teóricas y políticas 
que refuercen esencialismos sobre las categorías sociales e identitarias de estos colectivos.

Por otra parte, el sistema capitalista $y$ patriarcal genera discriminaciones en razón de las capacidades físicas e intelectuales de las personas. Bajo la lógica de exclusión de dichos sistemas, se parte de un modelo de hombre sin condiciones aparentes de discapacidad, que intervengan en su producción como mano de obra para el mercado.

El enfoque del Modelo Social de la Discapacidad expuesto por González (2010), explica que las discapacidades no pueden verse como limitaciones o deficiencias, sino que es el entorno el que reproduce desventajas para las personas que no son consideradas sanas o capaces, ya que se concibe una sociedad construida para $y$ por sujetos que no presentan ninguna discapacidad.

De esta forma, es claro que las diferencias por sí mismas no provocan desigualdades, sino que la exclusión es el resultado de acuerdos sociales y culturales que han sido institucionalizados por los grupos de poder o élites, que justifican y perpetúan manifestaciones de discriminación dirigidas hacia personas con características valoradas socialmente en forma negativa. Dicha construcción de lo que se considera diferente e inferior se da en el marco de sistemas históricos, como lo son el patriarcado, el capitalismo y el racismo, los cuales tienen como fin perpetuar relaciones de poder que garantizan la reproducción de los intereses económicos y políticos de dichos grupos dominantes en una sociedad dada.

\section{METODOLOGÍA}

La investigación en la cual se basa el presente artículo se desarrolló con el objetivo de analizar las manifestaciones de discriminación según género, etnia-raza, discapacidad y orientación del deseo sexual presentes en el ámbito universitario, según la percepción del estudiantado de la UNA. El estudio se efectuó desde los enfoques cuantitativo y cualitativo, recurriendo a diferentes técnicas, con el fin de tener una visión más amplia de la problemática estudiada. No obstante, para la realización del presente artículo se toman como base los resultados del enfoque cualitativo.

El estudio comprendió el periodo de enero de 2017 a junio de 2019. En el 2017, se inició con una revisión bibliográfica de investigaciones llevadas a cabo tanto a nivel nacional como internacional acerca de la temática en cuestión. Asimismo, se hizo una revisión exhaustiva sobre la normativa nacional e institucional que sustenta las acciones que se llevan a cabo en la UNA para la atención de la problemática y la documentación referida a los programas o acciones que se desarrollan en la institución para la promoción de la no discriminación. Ese mismo año se efectuaron 10 entrevistas semiestructuradas a personal de la UNA a cargo de implementar acciones de prevención $y$ de atención de la población estudiantil y se aplicó un cuestionario ${ }^{2}$ a una muestra probabilística de estudiantes del Campus Omar Dengo y del Campus Benjamín Núñez de la UNA.

Finalmente, para profundizar sobre las percepciones $y$ vivencias de las manifestaciones de discriminación en la población estudiantil, se desarrollaron cuatro grupos focales: 2 en el Campus Omar Dengo, 1 en el Campus Nicoya $y$ 1 en el Campus Liberia ${ }^{3}$. Se contó con la participación de un total de 16 estudiantes que cursan diferentes carreras en la UNA (10 mujeres y 6 hombres):

2 El cuestionario se diseñó con el fin de conocer las percepciones del estudiantado en torno a las manifestaciones de discriminación por razón de género, discapacidad, etnia-raza y orientación del deseo sexual, considerando el sexo como variable transversal. Se aplicó a una muestra de 444 estudiantes, la cual contó con un $95 \%$ de confianza y un $5 \%$ de error máximo, utilizando el muestreo por conglomerados. Para la selección de las personas participantes se utilizó como marco muestral un listado con los cursos impartidos en el II ciclo lectivo del año 2017 y la cantidad de personas estudiantes matriculadas en cada uno. Los datos obtenidos a partir de la muestra seleccionada fueron expandidos para fines del análisis de resultados.

3 Se reconoce la importancia de realizar futuras investigaciones a nivel de otras sedes de la UNA, ya que, de acuerdo con su ubicación y contexto particular, se podrían presentar resultados que difieran de los campus seleccionados. 
$\diamond \quad 5$ indígenas (2 de los territorios NgobeBugle de Conte Burica y Osa, 3 del Territorio Indígena de Matambú)

$\diamond \quad 3$ con discapacidad

$\diamond \quad 6$ con diferentes orientaciones del deseo sexual con respecto a la heterosexualidad (1 lesbiana, 3 homosexuales y 2 bisexuales) $\diamond \quad 2$ afrodescendientes

La mayoría con edades entre los 18 y 30 años, excepto una estudiante cuya edad supera los 50 años. Además, 2 mujeres con discapacidad y 2 mujeres bisexuales son madres. Se pretendía la participación de estudiantes de todos los grupos de interés en cada grupo focal, sin embargo, esto no fue posible ${ }^{4}$. El estudiantado fue referido por algunas de las instancias universitarias citadas al inicio de este artículo, que trabajan con estas poblaciones, o bien, por otras personas estudiantes contactadas, mediante el muestreo en cadena o bola de nieve.

Para llevar a cabo los grupos focales se diseñó una guía con los aspectos que orientaron la discusión de la temática con el estudiantado participante y que abarcó sus concepciones sobre la discriminación, sus vivencias en el campus universitario y las recomendaciones que hacen a la institución para erradicar esta problemática. Asimismo, previo al inicio de cada grupo focal, se firmó un consentimiento informado con el fin de resguardar la participación de las personas y garantizar la confidencialidad. De igual manera, se solicitó la autorización respectiva para grabar el audio de cada actividad, con el fin de transcribir posteriormente la información. Cada grupo focal tuvo una duración de 2 horas aproximadamente. Con base en la información obtenida en los grupos focales, se desarrollan a continuación los principales resultados y su discusión respectiva.

Si bien, se convocó a población asiática, no se contó con su asistencia. No obstante, se reconoce la importancia de profundizar en la percepción y vivencia de la discriminación de este grupo poblacional dentro del ámbito universitario.

\section{RESULTADOS Y DISCUSIÓN}

La discriminación se manifiesta a través del desprecio que responde a un prejuicio o estigma social, expresado por medio del lenguaje verbal y simbólico, de acciones $u$ omisiones, con efectos que pueden ser intencionales o no, de limitar, coartar o suspender derechos y libertades de las personas que se encuentran en desventaja social (Rodríguez, 2004).

La investigación realizada muestra cómo la orientación del deseo sexual se convierte en un marcador de diferencia $y$ en un motivo de estigmatización y exclusión dentro del espacio universitario. Dentro de las vivencias expresadas por parte del estudiantado que participó de los grupos focales, se encuentra el haber presenciado, $y$ algunas veces experimentado, comentarios y miradas despectivas, reflejando el rechazo hacia esta población. Un ejemplo claro de esto es el comentario que aporta una persona participante: "pasaron unos muchachos y le iban diciendo cosas a otro muchacho que es gay, le decían que era un maricón, que los gays no son hombres" (E4, Grupo Focal, 15/03/2018, Heredia).

Maffia (2006) expone que existen procesos de definición e identificación, unidos a una reacción social que por lo general va acompañado de una distribución desigual de poder, en la cual se establece quién y desde qué lugar nombra, rotula, excluye y provoca. Puertas (2004) agrega que los estereotipos tienen una función social que busca dar explicación y racionalizar hechos sociales, al buscar mantener la identidad del grupo de pertenencia por encima del grupo que está siendo discriminado, por lo que comentarios como el mencionado anteriormente, buscan normalizar a quien es identificado como diferente, violentando sus derechos fundamentales.

Otra forma discriminatoria que refiere el estudiantado sexualmente diverso participante de los grupos focales es el trato diferenciado y las conductas que son sancionadas en parejas del mismo sexo y que no son señaladas de la misma forma en parejas heterosexuales. Esto lo explica uno de los estudiantes: “... cuando estaba yo con un chico como por el hueco (hondonada) [...] los guardias siempre que llegaban, 
a los primeros que llegaban eran donde nosotros y el resto de las parejillas que eran hombre y mujer ahí los dejaban" (E2, Grupo Focal, 15/03/2018, Heredia).

Careaga (2004) explica que hay una demanda social en donde las parejas heterosexuales pueden compartir espacios en común con amistades, familia y sociedad, pero a las personas lesbianas, gay y bisexuales se les exige mantenerse en espacios exclusivos y ocultos, bajo la amenaza permanente del rechazo social y la marginación; en palabras de la autora "se les permite estar, pero sin ser" (p.11).

Asimismo, dentro de los relatos, se hace referencia a situaciones que expresan la presencia de violencia simbólica, ya que tanto estudiantes del Campus Omar Dengo como del Campus Liberia mencionaron la ausencia intermitente de la Bandera de la Diversidad en las plazas respectivas, la cual se coloca en fechas particulares, pero se retira sin mayor justificación. Incluso, en el caso del Campus Liberia, refieren que para la Semana Universitaria del 2017, se izó la bandera, no obstante, tres días después la encontraron en la basura (E2, Grupo Focal, 16/05/2018, Liberia).

Cuando se habla de violencia simbólica se hace referencia a aquellas acciones coercitivas que establecen costumbres sociales basadas en expectativas colectivas, sutiles, casi imperceptibles que hacen que actos discriminatorios sean vistos como normales o naturales (Bourdieu, 1999, citado por Fernández, 2005).

Ante esto, es claro que el trato diferenciado en el que se niegan derechos a un grupo social puede darse tanto por acción como por omisión. El no hacer ni decir nada para evitar estas acciones discriminatorias, refuerza la exclusión, haciendo que se convierta en una acción que violenta y vulnera el desarrollo de las personas que son excluidas.

En la misma línea, el estudiantado relata la existencia de un cuestionamiento constante hacia las personas homosexuales que practican una religión, ya que se les señala como una contradicción sus creencias religiosas con respecto a sus prácticas sexuales y afectivas, lo cual reclaman como un derecho que se está violentando, a partir de un discurso normalizado por algunas personas dentro de la universidad (E3, Grupo Focal, 16/05/2018, Liberia).

Pecheny (2001) afirma que es un hecho que estas acciones favorecen el mantenimiento de prejuicios y estereotipos, el aumento de actos violentos hacia esta población, así como, una mayor exposición a infecciones de transmisión sexual, entre otras consecuencias. Lo anterior, ya que se está limitando el derecho al libre tránsito, a la educación sexual y a la educación en general, a la salud, al libre culto, entre otros.

En cuanto a las personas con discapacidad, su incorporación a la educación es un derecho humano que ya ha sido ratificado a través de una serie de convenciones internacionales y de la legislación nacional, la UNA no ha estado ajena a estos esfuerzos. No obstante, la población con discapacidad que participó de los grupos focales menciona haberse enfrentado a barreras actitudinales $y$ de infraestructura que limitan su desarrollo educativo, incluso, al llevar a que parte del estudiantado con estas condiciones tenga que abandonar cursos o la universidad. Un estudiante con discapacidad compartió su experiencia:

Cuando esa profesora me volvió a ver con el bastón... se queda como... ¿Qué voy a hacer yo con usted?... vea - dice- Yo no puedo, mire, yo tengo que sacar 30 minutos para explicarles a ellos $y$ después el resto dedicarme a usted, yo no sé qué voy a hacer si usted ni siquiera sabe escribir (E4, Grupo Focal, 15/03/2018, Heredia).

El reconocimiento del estudiantado con sus diversas condiciones exige que el personal docente cuente con herramientas actitudinales $y$ de formación para generar en el aula estrategias metodológicas y evaluativas que garanticen ambientes de aprendizaje inclusivos y acordes con las necesidades específicas de las personas.

Otra de las limitaciones a las que hizo alusión el estudiantado en condición de discapacidad que participó en los grupos focales, fue las barreras que persisten en lo referido a la infraestructura universitaria, ya que se han encontrado con problemas como: falta de losetas; 
rampas demasiado inclinadas o que el material con las que están diseñadas es inadecuado, ya que son resbalosas o provocan vibración con el paso de la silla de ruedas; $y$ edificios antiguos que son completamente inaccesibles. Al respecto, una estudiante con discapacidad participante de la investigación comentó:

...la universidad está construyendo nuevos edificios y está procurando mejorar todo, pero lo hace desde una visión de $\mathrm{x} 0$ $y$ ingeniero, $y$ el ingeniero no es una persona con discapacidad, por lo tanto, no tiene el requerimiento de la necesidad, ni sabe cómo sí o cómo no (E1, Grupo Focal, 15/03/2018, Heredia).

Magendzo (2000) expone que el derecho a la educación va más allá de facilitar el ingreso a los espacios educativos sin restricciones, sino que exige la creación de condiciones para que la población que se busca beneficiar permanezca y se egrese de forma exitosa del sistema educacional.

Lo anterior se ve limitado cuando no se toma en consideración el contexto social de la población que se quiere beneficiar, en este caso el estudiantado que presenta condiciones diversas de discapacidad, ya que como se rescata de la información obtenida de los grupos focales, el acceso a la tecnología ha sido vista como un avance y una herramienta que busca facilitar y mejorar el acceso al conocimiento. No obstante, para las personas con discapacidad, el establecer trámites tecnológicos no siempre representan una ventaja. Quienes participaron de los diferentes grupos, exponen que trámites específicos como el uso del Aula Virtual, solicitud de becas, matrícula, entre otros, genera una limitante para quienes cuentan con una discapacidad visual, ya que como lo expone una de las estudiantes: “... las páginas están hechas solo para gente que ve... porque no piensan en que nosotros utilizamos programas, que utilizamos este... medios, recursos para poder, di pues, estudiar" (E5, Grupo Focal, 15/03/2018, Heredia).

El enfoque de interseccionalidad explica que estas limitaciones que genera el contexto social se agudizan cuando convergen en una misma persona una o más condiciones, que en el marco de sociedades patriarcales $y$ capitalistas, son rechazadas o construidas ideológicamente como inferiores. Un ejemplo de esto es la vivencia de una de las estudiantes que tiene una discapacidad visual y que además es madre:

Yo llamé... y ahí hablé con una señora ...Yo le expliqué, le digo: Vea señora es que yo estoy viviendo muy largo, estoy con mi hija y me dice - ¿Y nosotros que culpa tenemos de que usted tenga una hija? - diay, dice - Imagínese si usted apenas lleva tres cursos ¿De qué vale que venga a la universidad (E5, Grupo Focal, 15/03/2018, Heredia).

Desde el enfoque de interseccionalidad se señala que la confluencia de dos o más factores de vulnerabilidad favorece una múltiple discriminación, lo que implica reconocer especificidades en la forma como se vive la exclusión si se es mujer u hombre con discapacidad, aunado a otros factores como la clase social, la etnia, si se tiene o no hijos o hijas, entre otros aspectos.

Esta múltiple discriminación también la apuntó una estudiante con discapacidad motora, quien por su mediana edad ha sido aún más excluida, si bien, no tuvo problemas para ingresar a la universidad, se ha enfrentado a dinámicas que suponen que tiene todo el conocimiento tecnológico para hacer uso de los sistemas informáticos institucionales, al igual que una persona adulta joven de zona urbana que viene saliendo del colegio (E1, Grupo Focal, 15/03/2018, Heredia).

González (2010) explica que condiciones como la raza, el origen nacional o étnico, el sexo, la orientación sexual, la identidad de género, la edad, la discapacidad, la religión, la condición socioeconómica u otras condiciones, se convierten en capas de desigualdad que interactúan en forma simultánea en una misma persona, por lo que identidades distintas pueden representar sistemas de opresión o privilegio dependiendo del contexto.

En esta misma línea, la población afrodescendiente e indígena que participó en los grupos focales, señaló que ha recibido discriminación por su color de piel y su origen étnico 
a través de la exclusión de grupos para realizar trabajos de cursos; así mismo, la solicitud constante de demostrar qué tanto son afrodescendientes o indígenas, a partir de referencias respecto a su idioma o alimentación; también apuntaron haber recibido comentarios despectivos hacia su cuerpo. Al respecto, una estudiante afrodescendiente comentó: “...recuerdo que yo llegué a una general y llegué tempranísimo y lo primero que escuché fue decir: - Uy una negra, tenemos una negra en la clase" (E1, Grupo Focal, 10/05/2018, Heredia).

La cosificación de las personas a partir de sus rasgos físicos, sus corporalidades y su cultura, refuerza la existencia de prejuicios que se construyen desde posiciones de privilegio social, en este caso de personas que se asumen blancas y no indígenas, lo cual, coincide con la vivencia de otra de las estudiantes que afirma que fue comparada con Pocahontas ${ }^{5}$ debido a su color de piel, por parte de una profesora (E2, Grupo Focal, 16/05/2018, Liberia).

Asimismo, otra estudiante agregó: "Entonces otra parte de discriminación que yo puedo sufrir... es por ejemplo en el idioma, porque entonces a mí me dicen: Ah bueno, entonces hábleme para ver si es cierto" (E3, Grupo Focal, 10/05/2018, Heredia). Para Magendzo (2000), estas afirmaciones se realizan dentro de un acto descalificador y desvalorizante del bagaje cultural y de la identidad de las personas que están siendo discriminadas.

Las vivencias anteriores son compartidas por otras estudiantes indígenas, quienes incluso afirman que compañeras han negado su procedencia con el fin de evitar actos de discriminación, mientras que otras han tenido que enfrentar situaciones de hostigamiento sexual, donde el hecho de ser indígena $y$ mujer les ha colocado en un espacio de mayor vulnerabilidad para recibir esta forma de violencia (E3, Grupo Focal, 10/05/2018, Heredia). La misma estudiante comentó que también ha sido objeto de comentarios despectivos $y$

5 Princesa indígena de la tribu Powhatan del siglo XVII, la cual es retratada en el personaje Pocahontas en la película de dibujos animados de Disney. violentos por parte de docentes, compartiendo una de sus experiencias:

... hizo una comparación sumamente agresiva, dijo que en esa época los indígenas $y$ todas las poblaciones que existían debían ser sumisos a los españoles por lo que estaba ocurriendo... entonces dice: -Es como el caso entre la compañera y yo, entonces ella me debe respeto, me debe... debe ser sumisa... (E3, Grupo Focal, 10/05/2018, Heredia).

Según Flores (2005), el sistema educativo es uno de los espacios que más influye en la constitución de la identidad de las personas y en la construcción de su plan de vida, "se construyen un conjunto de rasgos de pensamiento, de valoraciones, de afectos, de actitudes y comportamientos que se asumen como típicos y como referentes del deber ser y de pertenencia" (p. 73). Por lo tanto, toda práctica o discurso que tenga como consecuencia la exclusión o el rechazo de las diversidades e identidades que caracterizan al estudiantado, refuerzan relaciones de poder $y$ de dominio. Se constituyen en un currículo oculto, que al ser permitido y pasar desapercibido por las personas que lo atestiguan (pero no lo confrontan), así como por parte de quienes lo ejercen, se convierte en norma y de esta forma se perpetúan relaciones de desigualdad.

Al igual que la población con discapacidad, la población indígena refirió que el acceso a la tecnología puede convertirse en una forma de exclusión. Si bien, la UNA cuenta con una serie de acciones para facilitar el ingreso académico de estudiantes indígenas, el tener que enfrentarse a los trámites habituales que se realizan por medio de internet, tales como, la solicitud de becas, la matrícula, las citas médicas, el Aula Virtual, entre otras, se constituye en una barrera que amenaza su continuidad dentro de la universidad. Esto queda aún más claro con la siguiente vivencia de un estudiante del Territorio Indígena de Matambú: “... cómo quiere que yo entre a la computadora si no sé cómo se enciende, en mi casa no hay luz, en mi casa no existe la electricidad, menos el internet, 
menos una computadora" (E5, Grupo Focal, 15/05/2018, Nicoya).

Ante esto, reviste importancia lo expuesto por González (2010), quien hace referencia a que la discriminación no solo incluye los actos en sí mismos, sino también la omisión de acciones necesarias para que todas las personas tengan el mismo acceso en las mejores condiciones - en este caso a la educación superior-, por lo que afirma que la discriminación puede ser ejercida tanto de manera consciente como inconsciente, pero los resultados son los que finalmente dan cuenta de su existencia.

El estudiantado indígena que participó de la investigación también expresó su inconformidad ante prácticas institucionales en las cuales no se les toma en cuenta su opinión, a pesar de que estas acciones parten del supuesto de que beneficiarán a estas poblaciones. Por ejemplo, señalaron que en ocasiones se trae a la universidad a indígenas provenientes de otros países, pero no se dan espacios para que indígenas del territorio costarricense compartan sus conocimientos; además, el hecho de que cursos sobre lenguas indígenas sean impartidos por personas no indígenas. También indicaron que se ha nombrado oficialmente a espacios universitarios usando términos de origen indígena, lo cual se hizo sin consultarles y lo valoran como un irrespeto, dado que para la población Bribrí, el nombre se asigna a partir de herencias culturales, no por medio de decisiones al azar.

Como se indicó anteriormente, el marcador género fue un eje transversal en la investigación en la que se basa este artículo. Al respecto, una de las situaciones que sobresalió en los grupos focales fue la condición de maternidad de las estudiantes, considerada como una limitante y cómo esta repercute en forma distinta en el desempeño académico en relación con los estudiantes que son padres.

Las mujeres participantes también hicieron referencia a que realizan tareas de cuido más allá de su condición de maternidad, en donde se refuerza a nivel social como un hecho "natural" que se hagan cargo de las necesidades de sus hijos e hijas y además de otras personas familiares, como lo son adultas mayores, con discapacidad, con alguna enfermedad, entre otras. Un ejemplo de esto lo expone una estudiante madre quien comenta que tiene dos hijos y que su hermana la apoya con el cuido de estos: “... ayer yo perdí digámosle el quiz, hablé con la profesora le digo: - No puedo ir, porque mi mamá está enferma, mi cuñado tuvo un accidente" (E5, Grupo Focal, 15/05/2018, Nicoya). Esta situación provocó que su hermana tuviera que asistir al hospital, por lo que la estudiante tuvo que hacerse cargo del cuido de sus hijos, sus sobrinos y de su madre enferma. La misma estudiante agrega que esta situación familiar ha sido un impedimento para realizar trabajos grupales. Ante esto, Arvizu y Miller (2016) exponen la necesidad de comprender que muchas de las estudiantes que asisten a la universidad "tienen una condición que les lleva a planificar de forma distinta su vida dentro y fuera de los espacios universitarios, pues deben atender responsabilidades con sus estudios y con sus familias" (pp. 25-26).

Una mirada interseccional en este análisis es indispensable, ya que condiciones que se traslapan como el hecho de ser mujer, madre $y$ de zona rural, dificultan el acceso a la educación, lo cual favorece la subordinación interseccional. De acuerdo con Muñoz (2011) ocurre cuando un factor discriminatorio confluye con otro que le antecede, aumentando el desempoderamiento.

Las estudiantes también señalaron que su condición de maternidad les obliga a que en muchas ocasiones tengan que asistir a clases con sus hijos o hijas, recibiendo en algunos casos el rechazo de parte de docentes, quienes han realizado comentarios que van desde acusaciones misóginas, como el asumir que ellas quedaron embarazadas porque "no se cuidaron", hasta tacharlas de irresponsables, lo cual no ocurre con estudiantes que son padres. Una estudiante madre comenta: “... la primera vez que la traje fue como "Ah ahora hay dos niñas" y lo dijo, así como un tono como ¿Por qué la trae?" (E4, Grupo Focal, 15/05/2018, Nicoya).

Bourdieu (1999, citado por Fernández, 2005) explica que la violencia simbólica también se presenta en la acción pedagógica que es parte de la educación formal, como bien lo representa la vivencia de esta estudiante. El autor 
explica que estos discursos terminan teniendo la misma función a nivel cultural que tiene la transmisión del capital genético en el ámbito de la biología, es decir, se vuelven parte de la cotidianidad al punto de pasar desapercibidos $y$ ser vistos como normales, e incluso, necesarios para mantener cierto orden social.

Otras de las necesidades señaladas es el disponer de cambiadores en los baños y salas de lactancia en todos los campus universitarios ya que, si bien, se cuenta con algunas salas, no todas presentan las condiciones adecuadas para amamantar a sus bebés. Asimismo, apuntaron la importancia de contar con alternativas de cuido de menores de edad dentro de las universidades, con el fin de favorecer que las estudiantes que son madres no deserten por esta causa y puedan graduarse exitosamente.

Darré, Sosa y Baramendi (2014) hacen el señalamiento de que, si se ofrece el mismo tratamiento a personas con diferentes realidades, se estaría acentuando una práctica que finalmente resultaría excluyente. Esto implica reconocer que las estudiantes que son madres están en clara desventaja frente a sus compañeras que no lo son, así como frente a sus compañeros varones, ya sean padres o no.

De esta forma, no se puede ignorar que las personas con identidades que históricamente han sido subordinadas, $y$ en las cuales convergen diversos factores de exclusión, se enfrentan a formas de discriminación que van desde lo sutil a expresiones violentas y fácilmente reconocibles, por lo que si se quieren eliminar estas situaciones dentro de los espacios universitarios debe iniciarse por nombrar las manifestaciones, identificarlas y tomar acciones urgentes para su erradicación.

\section{CONCLUSIONES Y RECOMENDACIONES}

La discriminación social se sustenta en el establecimiento de jerarquías que consolidan el poder hegemónico de grupos privilegiados sobre otras poblaciones que han sido históricamente vulnerabilizadas. Tener un acercamiento a esta problemática desde el enfoque de género $y$ el enfoque interseccional permite entender que categorías como la etnia, la raza, el sexo, el género, la orientación del deseo sexual, las capacidades, entre otras condiciones, pueden significar categorías de privilegio o de limitaciones, dependiendo del contexto donde cada persona se desarrolle, negándole a las personas que son discriminadas, el cumplimiento de sus derechos humanos, la restricción de su libertad y el acceso a oportunidades (Munévar y Mena, 2009 y 2013).

Con base en lo anterior, dentro de las principales conclusiones de esta investigación, destaca el reconocimiento de que la unA ha promovido desde su fundación acciones dirigidas a la inclusión de sectores sociales con mayor desventaja social, así como a la prevención y atención de situaciones de violencia y de discriminación. Sobresale la aprobación e implementación de la Política contra el Hostigamiento Sexual (2009) y su respectivo reglamento, la Política para la Igualdad y Equidad de Género en la UNA (PIEG-UNA) (2010) y la Declaratoria de la UNA como espacio libre de todo tipo de discriminación (aprobada en el 2011 y ampliada en el 2015). Asimismo, sobresale la creación del Instituto de Estudios de la Mujer en el año 1991, como unidad académica especializada en el abordaje de temas que involucran el desarrollo y bienestar de las mujeres y otros grupos históricamente vulnerabilizados; así como otras instancias que trabajan en forma directa en la defensa de los derechos de la población estudiantil, como lo son la Defensoría Estudiantil y la Federación de Estudiantes de la UNA.

Los resultados evidencian que parte de la población universitaria reproduce estereotipos $y$ prejuicios hacia las personas que no son heterosexuales, lo cual se refleja en las vivencias del estudiantado participante de los grupos focales, que refiere recibir trato diferenciado, burlas, chistes, miradas o gestos ante lo considerado socialmente inaceptable o anormal, hasta actos que pueden ser analizados como expresiones de violencia simbólica, tales como, la ausencia y desaparición de la Bandera de la Diversidad dentro de los espacios universitarios.

Por su parte, el estudiantado con discapacidad delimitó tres ejes centrales de exclusión. El primero refiere a factores actitudinales $y$ de falta de formación por parte del personal docente $y$ del sector estudiantil, en lo que se 
refiere a la atención o acompañamiento de estudiantes con diversas condiciones de discapacidad. El segundo eje hace hincapié en la infraestructura, la cual no es accesible en toda la universidad, siendo necesario que se garantice que las personas con diversos tipos de discapacidad puedan desplazarse libremente en los distintos campus universitarios. El tercer eje señala la necesidad de que la totalidad de los sistemas informáticos de la institución sean accesibles a las personas con discapacidad visual.

Del mismo modo, poblaciones como la indígena $y$ afrodescendiente también han señalado la presencia de manifestaciones de discriminación dentro de la universidad. Sobresale la necesidad de visibilizar a todos los colectivos que conforman la población universitaria, así como el reconocer sus aportes, necesidades $y$ vivencias. Una de las formas en que la población indígena vive la discriminación es a través del uso de los sistemas informáticos, al considerar de que parte de la tramitología universitaria se realiza a través de internet y algunos estudiantes no tiene acceso, ni conoce el funcionamiento de estos sistemas.

Por otra parte, las conductas sexistas siguen estando presentes en el ámbito universitario a través de vivencias como la exclusión de actividades, hostigamiento sexual, descalificación del conocimiento de las mujeres, comentarios ofensivos por dicha condición, entre otros. Las formas y mecanismos de exclusión se agudizan cuando se entrecruzan con otras condiciones como el ser madre, provenir de zona rural y tener una condición socioeconómica de bajos recursos.

Lo anterior señala la necesidad de realizar acciones efectivas e inmediatas para la eliminación de toda forma de discriminación dentro del espacio universitario. En el caso particular de la UNA, esto cobra mayor relevancia, ya que su fundación —en 1973 - responde a la necesidad de implementar un modelo de desarrollo integral e incluyente, dirigido a los sectores sociales de la población costarricense menos favorecidos y en condición de mayor vulnerabilidad (UNA, 2016). Por lo tanto, las acciones que se dirijan a estos grupos no solo deben perseguir su inclusión en el ámbito universitario, sino también su permanencia en condiciones óptimas, la cual se ve severamente amenazada por cualquier forma de discriminación.

Se recomienda que toda acción institucional que busque el beneficio para algún grupo universitario históricamente vulnerabilizado, incluya una consulta participativa a dicha población, de lo contrario, terminarán siendo ajenas a su grupo meta e incluso pueden continuar siendo igualmente excluyentes. Asimismo, se recomienda ampliar la capacitación a toda la comunidad universitaria en materia de prevención, atención y eliminación de la discriminación, así como fortalecer las instancias responsables de la atención de las distintas formas de discriminación dentro de la universidad.

Finalmente, se requiere promover el compromiso ético y político de la población universitaria con el fin de que vivir libre de toda forma de discriminación se reconozca y garantice como un derecho fundamental para el desarrollo social y a nivel de la educación superior, así como, la comprensión de que la erradicación de esta problemática es una tarea cotidiana que involucra a todas las personas que conforman la comunidad universitaria.

\section{REFERENCIAS}

Arvizu, V. y Miller, D. (2016). Ser madre y estudiante. Una exploración de las características de las universitarias con hijos y breves notas para su estudio. Revista de la Educación Superior. México: Universidad Autónoma Metropolitana, Azcapotzalco.

Campbell, E. (2002). Desafiando poderes. Sexismo y racismo: un acercamiento desde las mujeres afrodescendientes". En E. Campbell y G. Careaga (Eds.), Poderes cuestionados: Sexismo y racismo en América Latina, 1.ed. San José, Costa Rica: Diseño Editorial.

Careaga, G. (2004). Sexualidades diversas: Aproximaciones para su análisis. Programa Universitario de Estudios de Género (PUEG), México: Universidad Universidad Nacional Autónoma de México.

Darré, S., Sosa, F. y Baramendi, C. (2014). Diversidad etaria y educación superior. 
Guía para acompañar procesos de sensibilización sobre inclusión social y equidad desde la perspectiva interseccional. San José, Costa Rica: Instituto de Estudios de la Mujer, Universidad Nacional.

Fernández, J. (2005). La noción de Violencia Simbólica en la obra de Pierre Bourdieu: Una aproximación crítica. Cuadernos de Trabajo Social, 18, 7-31.

Flores, R. (2005). Violencia de género en la escuela: sus efectos en la identidad, en la autoestima y en el proyecto de vida. Revista Iberoamericana de Educación, (38).

González, R. (setiembre de 2010). Las mujeres con discapacidad y sus múltiples desigualdades: Un colectivo todavía invisibilizado en los estados latinoamericanos $y$ en las agencias de cooperación internacional. XIV Encuentro de Latinoamericanistas Españoles: Congreso Internacional (pp.2737-2756). Santiago de Compostela, España.

Lamas, M. (mayo de 2002). Cuerpo: diferencia sexual y género. México: Taurus, Alfaguara.

Maffia, D. (2006). Lo que no tiene nombre. CONAPRED: Disidencia sexual e identidades sexuales y genéricas. México, D.F, México: Consejo Nacional para Prevenir la Discriminación.

Magendzo, K. (julio de 2000). La diversidad y la no discriminación: Un desafío para una educación moderna. Pensamiento Educativo, 26, 173-200. Recuperado de http://www.pensamientoeducativo.uc.cl/ files/journals/2/articles/171/public/171417-1-PB.pdf

Meler, I. (1998). La familia, antecedentes históricos y perspectivas futuras. En M. Burin e I. Meler, Género y familia. Poder, amor y sexualidad en la construcción de la subjetividad. Argentina: Editorial Paidós.

Munévar, D. y Mena, L. (2009). Violencia estructural de Género. Revista Facultad de Medicina, (57), 356-365.

Munévar, D. y Mena, L. (2013). Mujeres afrodescendientes en situación de discapacidad.
Diálogos interseccionales en la academia, Universidad Nacional de Colombia, Colombia.

Muñoz, P. (2011). Violencias Interseccionales Debates Feministas y Marcos Teóricos en el tema de Pobreza y Violencia contra las Mujeres en Latinoamérica. Central America Women's Network (CAWN), Honduras.

Organización de los Estados Americanos. (2019). Convención Interamericana contra toda forma de Discriminación e Intolerancia. Recuperado de http://www. oas.org/es/sla/ddi/tratados_multilaterales_interamericanos_A-69_discriminacion_intolerancia.asp

Organización de los Estados Americanos. (2019). Costa Rica firmó en la OEA la Convención Interamericana contra Toda Forma de Discriminación e Intolerancia. Recuperado de http://www. oas.org/es/centro_noticias/fotonoticia. asp? SCodigo=FNC-96425

Pecheny, M. (2001). De la "no discriminación" al "reconocimiento social". Un análisis de la evolución de las demandas políticas de las minorías sexuales en América Latina. XXIII Congreso de la Latin American Association, Washinton D.C., EUA.

Puertas, S. (2004). Aspectos teóricos sobre el estereotipo, el prejuicio y la discriminación. Seminario Médico, 56, 134-144.

Rodríguez, J. (2004). ¿Qué es la discriminación y cómo combatirla? México, D.F, México. Recuperado de http://repositorio.dpe.gob.ec/bitstream/39000/950/1/ CONAPRED-037.pdf

Saltzman, J. (1992). Equidad y Género. España: Ediciones Cátedra.

Segato, R. L. (2006.) Racismo, Discriminación $y$ acciones afirmativas: Herramientas conceptuales. Serie Antropológica. Brasil: Universidad de Brasilia.

Universidad Nacional de Costa Rica (UNA). (20 de octubre de 2009). Política contra el Hostigamiento Sexual en la Universidad Nacional. UNA-Gaceta 17-2009. Heredia, Costa Rica: Universidad Nacional de Costa Rica.

Universidad Nacional de Costa Rica (UNA). (22 de febrero de 2016,). Estatuto Orgánico de la Universidad Nacional. UNA-Gaceta 
Extraordinaria nro. 3-2016. Heredia, Costa Rica.

Universidad Nacional de Costa Rica (UNA). (20 de octubre de 2009). Reglamento para Prevenir, Investigar y Sancionar el Hostigamiento Sexual en la Universidad Nacional. UNA- Gaceta 17-2009. Heredia, Costa Rica.

Universidad Nacional de Costa Rica (UNA). (21 de mayo de 2010). Política para la Igualdad y Equidad de Género en la Universidad Nacional. Gaceta UNA-9-2010. Heredia, Costa Rica.

Universidad Nacional de Costa Rica (UNA). (11 de agosto de 2011). Acuerdo SCU-1519-2011
Universidad libre de todo tipo de discriminación. Acta nro. 3176. Costa Rica.

Universidad Nacional de Costa Rica (UNA). (16 de setiembre de 2015). Modificación del acuerdo scu-1519-2011 del 12 de agosto del 2010 sobre el tema de la Universidad libre de todo tipo de discriminación. UNA-Gaceta Ordinaria nro. 17. Heredia, Costa Rica.

Fecha de ingreso: 20/06/2019 Fecha de aprobación: 23/09/2019 